\title{
I prezzi del latte in Europa: uno studio comparato
}

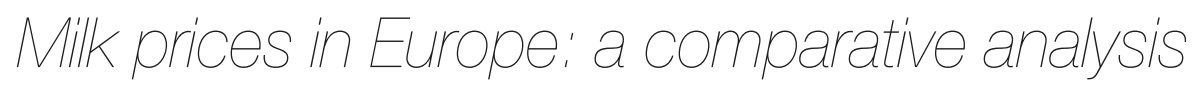

${ }^{1}$ Fondazione CRPA Studi e Ricerche, Reggio Emilia, Italy. ${ }^{2}$ ZuiveINL, Den Haag, The Netherlands

\author{
${ }^{*}$ Corresponding author: \\ Alberto Menghi \\ Fondazione CRPA, Viale Timavo 43/2 42100 Reggio Emilia \\ a.menghi@crpa.it
}

Ricevuto il 12 settembre 2019

Accettato il 6 ottobre 2019

DOI: $10.36138 / S T L C .01 .2019 .04$

\section{Riassunto}

II presente articolo riporta i risultati di un'analisi comparativa realizzata sui prezzi del latte in Europa e sulla loro composizione. Promossa a partire nel 1999 dall'organizzazione interprofessionale lattiero casearia olandese (Dutch Dairy Board) su mandato del sindacato agricolo olandese LTO e svolta in collaborazione con l'associazione di produttori European Dairy Farmers (EDF), questa indagine ha avuto lo scopo di aumentare la trasparenza del mercato del latte in Europa. I risultati permettono, inoltre, di capire i principali meccanismi di formazione del prezzo del latte e le componenti aggiuntive che negli ultimi anni stanno condizionando il modo di produrre latte. Un esempio molto interessante è la proliferazione di disciplinari produttivi sul tema della sostenibilità, molto diversi tra di loro ma che su base volontaria o obbligatoria devono essere sempre più rispettati dai produttori di latte per poter continuare a produrre.

\section{Parole chiave:}

Prezzo del latte

Mercati Agricoli

- Sostenibiltà

- Cooperative agricole

Produttori di latte

\section{INTRODUZIONE}

II presente studio ha lo scopo di presentare i risultati di un progetto volto ad aumentare la trasparenza del mercato del latte a livello europeo. Si tratta di uno studio che viene condotto a partire dal 1999 dal Dutch Dairy Board (Productschap Zuivel) l'organizzazione interprofessionale lattiero casearia olandese su mandato del sindacato agricolo olandese LTO e svolto in collaborazione con European Dairy Farmers (EDF) un'associazione di produttori latte europea. Lo

\section{Abstract}

This article reports the results of a comparative analysis carried out on milk prices in Europe and their composition. Promoted in 1999 by the Dutch Dairy Interprofessional Organization (Dutch Dairy Board) upon farmer's union LTO request and carried out in collaboration with the European Dairy Farmers (EDF) producer association, this survey was aimed at increasing the transparency of the milk market in Europe. The results also make it possible to understand the main milk price formation mechanisms and their additional components that have been influencing the way to produce milk in recent years. A very interesting example is the proliferation of production standards on the subject of sustainability, very different from each other but which on a voluntary or mandatory basis must be increasingly respected by milk producers to be able to continue producing milk.

\section{Keywords:}

Milk Price

Agricultural Markets

Sustainability

Agricultural Cooperatives

Milk Producers

scopo principale di questo studio è quello di aumentare la trasparenza del mercato del latte ed in particolare della formazione dei prezzi nei diversi Paesi europei. I risultati di queste analisi vengono pubblicati mensilmente e messi a disposizione di tutti gli operatori del settore. Inoltre, viene pubblicata una sintesi annuale che riassume l'andamento annuale dei prezzi rilevati.

Bisogna infine ricordare che questo progetto è nato in un periodo di scarsità di informazioni economiche comparative per il settore lat- 
tiero caseario europeo ed è stato precursore della nascita di altre fonti informative anche di carattere istituzionale come il Milk Market Observatory creato dalla Direzione Generale dell'Agricoltura della Commissione Europea (DG AGRI) solo di recente.

Secondo quanto teorizzato dagli economisti francesi Antoine-Augustine Cournot (1) e dal Leon Walras (2) è noto infatti che tra le caratteristiche di un "mercato perfetto" vi è la trasparenza del mercato o la perfetta informazione degli acquirenti e dei venditori sulla situazione del mercato. Questa assunzione assicura che in un regime di concorrenza ognuno sa quello che fanno gli altri per cui non si determinano opportunismi o tendenza ad imbrogliare gli altri operatori del mercato stesso (3).

La trasparenza riguarda solo uno degli aspetti cruciali di questo mercato caratterizzato da un elevato numero di produttori allevatori ma da un numero di acquirenti relativamente un limitato. Questo non permette a tutti gli operatori di essere dei "price taker" e cioè di pagare o riceve il prezzo generato dal mercato stesso in quanto nessuno degli operatori ha una quota di mercato tale da poter influenzare il prezzo stesso. I mercati agricoli per loro struttura hanno sempre manifestato dei limiti in termini di trasparenza, l'elevato numero di produttori spesso distribuiti in territori piuttosto ampi e l'elevato numero di acquirenti hanno sempre manifestato una carenza di trasparenza ha sempre reso difficile la realizzazione di un mercato perfetto così come veniva teorizzato dal Walras (4).

\section{MATERIALI E METODI}

La metodologia utilizzata consiste nel raccogliere mensilmente presso i produttori di latte conferenti a diverse industrie lattiero casearie le fatture che mensilmente vengono emesse nei confronti dell'acquirente, siano esse cooperative o soggetti privati. In genere si prediligono produttori che consegnano latte alle cooperative in quanto il rapporto di consegna è più stabile nel tempo rispetto a chi consegna a industrie private. In ambito europeo si è ritenuto utile inserire nel confronto anche i prezzi del latte della Svizzera anche se non appartenente all'Unione Europea e a completare il quadro del mercato del latte si è ritenuto inoltre utile inserire nella comparazione i prezzi di alcuni mercati particolarmente interessanti per il settore come gli Stati Uniti e la Nuova Zelanda.

Si tratta di uno studio che prosegue ininterrottamente da 20 anni. Nel corso degli anni si è avuto un continuo aggiornamento dei principali acquirenti operanti nel panorama europeo a seguito di fusioni, acquisizioni e disponibilità degli allevatori a fornire la documentazione necessaria all'analisi.

Nel 2018 sono stati raccolti dati di allevatori che hanno consegnato

\section{Tabella I: Latterie Europee analizzate nella presente analisi}

\begin{tabular}{|c|c|c|}
\hline 1 & Granarolo (Noord) & IT \\
\hline 2 & Valio & $\mathrm{Fl}$ \\
\hline 3 & FrieslandCampina & $\mathrm{NL}$ \\
\hline 4 & Arla Foods DK & $\mathrm{DK}$ \\
\hline 5 & Royal A-ware & $\mathrm{NL}$ \\
\hline 6 & Sodiaal (Pas de Calais) & FR \\
\hline 7 & Danone (Pas de Calais) & FR \\
\hline 8 & Lactalis (Pays de la Loire) & FR \\
\hline 9 & Hochwald Milch eG & $\mathrm{DE}$ \\
\hline 10 & Savencia (Basse Normandie) & FR \\
\hline 11 & Müller (Leppersdorf) & $\mathrm{DE}$ \\
\hline 12 & DMK Deutsches Milchkontor eG & $\mathrm{DE}$ \\
\hline 13 & Dairygold & $\mathrm{IE}$ \\
\hline 14 & Milcobel & $\mathrm{BE}$ \\
\hline 15 & Dairy Crest (Davidstow) & UK \\
\hline 16 & Glanbia & $\mathrm{IE}$ \\
\hline 17 & Kerry Agribusiness & $\mathrm{IE}$ \\
\hline
\end{tabular}

il latte a 17 diverse industrie lattiero casearie europee operanti in 9 Paesi (Italia, Finlandia, Danimarca, Olanda, Germania, Francia, Irlanda, Regno Unito, Belgio) (Tabella I).

Le fatture raccolte dai produttori di latte contengono i prezzi di vendita del latte risultanti da algoritmi di calcolo specifici e diversi per ciascuna industria lattiero casearia. Per rendere i risultati dei prezzi confrontabili viene effettuata una standardizzazione secondo i seguenti parametri di base:

- 4,2\% grasso;

3,4\% proteine;

conta batterica totale 24.999 ufc per ml;

conta delle cellule somatiche 249.999 ufc per ml;

consegna annuale 1.000.000 kg;

i prezzi sono definiti franco allevamento, considerando che la raccolta del latte avvenga ogni 2 giorni;

i prezzi sono calcolati IVA esclusa.

Questa standardizzazione che interessa tutti i dati raccolti non è sufficiente perché spesso intervengono altri elementi specifici che devono essere considerati: 
quando vengono utilizzati dei tempi di pagamento differenti (per esempio quindicinali) devono essere riportati al mese

- i prezzi vengono convertiti in Euro utilizzando un tasso di cambio medio mensile per quei Paesi dove la moneta unica non è stata adottata.

il volume è stato convertito in peso in quei contratti dove viene utilizzato il volume come unità di misura. Inoltre sono stati utilizzati i seguenti criteri di conversione: 1 litro equivale a 1,03 kg, 1 gallone equivale a 4,5461 litri e 1 cwt (USA) equivale a $45,36 \mathrm{~kg}$.

I dati confluiscono in un database che con le opportune standardizzazioni producono un report mensile che viene pubblicato nell'apposito sito web www.milkprices.com.

Oltre al prezzo calcolato e standardizzato, ciascuna industria lattiero casearia può utilizzare una serie di bonus o di penalizzazioni del prezzo che si sommano (o sottraggono) al valore di partenza andando a determinare il prezzo pagato all'allevatore. Anche questi elementi vengono considerati nel calcolo del prezzo mensile.

Non si può inoltre trascurare che per la maggior parte delle industrie lattiero casearie, ed in particolare per le cooperative, il prezzo mensile non rappresenta il prezzo finale che l'allevatore percepisce ma si tratta solo di un acconto che verrà poi confermato o rettificato a fine anno in base alla distribuzione dei profitti realizzati dalla cooperativa stessa a fine anno.

I pagamenti supplementari di conguaglio di fine anno vengono quindi inclusi nel prezzo del latte solo se sono direttamente collegati alla quantità di latte consegnato dal conferente e se vengono effettivamente distribuiti ai soci. Questo vuol dire che i dividendi dati ad azionisti che non consegnano latte o le somme destinate alla riserva della cooperativa vengono esclusi dalla formazione del prezzo del latte determinato in questo studio.

\section{IL PREZZO DEL LATTE E LA SOSTENIBILITÀ}

Un altro elemento che negli ultimi anni è andato ad influenzare il prezzo del latte in maniera sempre crescente, è dato dal tema della sostenibilità. Anche se non in modo non sempre sufficientemente chiaro, quasi tutte le industrie lattiero casearie hanno fatto proprio questo tema e hanno creato dei disciplinari di sostenibilità da far rispettare ai propri soci o ai propri conferenti. Gli elementi più comuni di questi disciplinari sono per esempio degli standard produttivi a livello di allevamento (migliore qualità del latte, protezione ambientale, benessere animale ecc.) In genere chi rispetta determinati parametri può ricevere un premio forfettario per unità di prodotto oppure possono essere creati dei sistemi di punteggio che possono variare da allevamento ad allevamento e in base a questi punteggi viene corrisposto il premio ad ogni singola azienda conferente.

Il premio di sostenibilità ha comunque lo scopo generale di far produrre un latte in modo sempre più sostenibile. Alcuni esempi sono di carattere ampio e applicati a livello nazionale e possono essere adottati da più aziende lattiero casearie: il Programma Nazionale per la Sostenibilità in Belgio (sustainability monitor), In Finlandia (NASEVA), in Irlanda (Sustainable Dairy Assurance Scheme - SDAS) e in Francia (La Charte des Bonnes Pratiques d'Elevage - CBPE), nel Regno Unito il British Red Tractor Scheme. Vi sono poi i disciplinari proposti dalle singole aziende in modo più specifico solamente ai propri conferenti. Per esempio, Arla (Arlagården Plus), DMK (Milkmaster), Hochwald (Milchplus), FrieslandCampina (Foqus planet), Royal A-ware (A-ware sustainability), Sodiaal (La Route du Lait (LRDL)), Dairy Crest (Davidstow Farm Standards), e Granarolo (Granlatte Strategic Plan 2018-2028).

Se la somma destinata ad incentivare la sostenibilità è nota da parte dell'azienda è possibile calcolarne l'incidenza dividendo questa somma per la quantità di latte prodotto in modo che si possa poi scorporare dal prezzo del latte pagato agli allevatori. Se questa somma non viene allocata in modo distinto è difficile scorporarla dal prezzo e quindi non può essere evidenziata in modo separato. In genere se non vi sono informazioni o se i premi di sostenibilità riguardano un limitato numero di produttori questo tipo di premio non viene indicato separatamente nel calcolo del prezzo fatto in questo studio. Questo viene fatto anche perché se la sostenibilità riguarda solo alcune linee di prodotto all'interno dell'azienda il risultato sarebbe difficilmente confrontabile con altre realtà e quindi verrebbe meno lo scopo dello studio stesso.

Una volta tenuto conto di tutti gli elementi sopra descritti è necessario cercare di individuare per ciascuna azienda lattiero casearia tra quelle considerate quali siano gli elementi che vanno a comporre il prezzo del latte perché ciascuna industria lattiero casearia calcola il prezzo del latte per i propri conferenti seguendo determinate regole interne.

\section{PREZZI DEI PAESI EXTRAEUROPEI}

Per avere un quadro più completo di confronto, si è ritenuto utile inserire nell'analisi di confronto anche due dei maggiori Paesi operanti sul mercato internazionale nell'ambito dell'import export dei prodotti lattiero caseari: Nuova Zelanda e Stati Uniti

In questo caso la metodologia non fa riferimento alla raccolta di fatture di soci conferenti a realtà produttive di questi Paesi ma è stato fatto riferimento a fonti ufficiali di pubblicazione dei prezzi. 


\section{Nuova Zelanda (Fonterra)}

II prezzo del latte calcolato per la Nuova Zelanda fa riferimento al prezzo pagato dalla maggiore cooperativa del Paese Fonterra ai propri soci conferenti. Il prezzo del latte viene pubblicato periodicamente dalla cooperativa stessa e include i conguagli di fine anno pagati ai soci conferenti della cooperativa. Oltre alle standardizzazioni relative alle unità di misura e al tasso di cambio tra Dollaro Neozelandese ed Euro è necessario operare una standardizzazione di tipo temporale. II prezzo del latte in Nuova Zelanda segue un ciclo annuale che va da giugno fino a maggio dell'anno successivo, per consentire un confronto con gli altri prezzi del latte riportati in questo studio è stato necessaria una conversione all'anno solare.

\section{Stati Uniti}

Come indicatore del prezzo del latte negli Stati Uniti è stato preso come riferimento il latte classe III pubblicato mensilmente dall'USDA valutato in dollari per Cwt (hundredweights) che corrisponde a $45,36 \mathrm{~kg}$.

II prezzo del latte pagato agli allevatori e pubblicato negli Stati Uniti nella maggior parte dei casi (circa l'80\%) fa riferimento al cosiddetto Federal Milk Marketing Orders (FMMOs). Questo FMMOs richiede alle industrie lattiero casearie di rendere pubblico quanto prodotto hanno venduto e a quale prezzo. Tramite degli specifici algoritmi questi prezzi e quantità vengono convertite in un prezzo minimo per il latte declinato però in 4 categorie di qualità del prodotto in

\section{Tabella II: Valori medi del prezzo del latte calcolati per le diverse Industrie lattiero casearie europee nel 2018 e nel 2017}

\begin{tabular}{|c|c|c|c|c|c|c|}
\hline Industrie lattiero casearie & Paese & $2018 € / 100 \mathrm{~kg}$ & $2017 € / 100 \mathrm{~kg}$ & $\begin{array}{c}\text { 2018-2017 Var. } \\
\text { Assoluta }\end{array}$ & 2018/2017 Var. \% & nat. Valuta \\
\hline Granarolo (Nord) & IT & 38,36 & 39,81 & $-1,45$ & $-3,6 \%$ & \\
\hline Valio & $\mathrm{Fl}$ & 36,74 & 36,64 & 0,10 & $0,3 \%$ & \\
\hline FrieslandCampina & $\mathrm{NL}$ & 36,07 & 38,56 & $-2,50$ & $-6,5 \%$ & \\
\hline Arla Foods DK & DK & 35,48 & 36,46 & $-0,98$ & $-2,7 \%$ & $-2,5 \%$ \\
\hline Royal A-ware & $\mathrm{NL}$ & 35,13 & 37,95 & $-2,82$ & $-7,4 \%$ & \\
\hline Sodiaal (Pas de Calais) & FR & 34,93 & 34,37 & 0,55 & $1,6 \%$ & \\
\hline Danone (Pas de Calais) & FR & 34,55 & 34,47 & 0,08 & $0,2 \%$ & \\
\hline Lactalis (Pays de la Loire) & FR & 33,98 & 33,55 & 0,43 & $1,3 \%$ & \\
\hline Hochwald Milch eG & $\mathrm{DE}$ & 33,74 & 34,08 & $-0,35$ & $-1,0 \%$ & \\
\hline Savencia (Basse Norman-die) & $\mathrm{FR}$ & 33,69 & 33,93 & $-0,24$ & $-0,7 \%$ & \\
\hline Müller (Leppersdorf) & $\mathrm{DE}$ & 33,02 & 35,38 & $-2,36$ & $-6,7 \%$ & \\
\hline DMK Deutsches Milchkon-tor eG & $\mathrm{DE}$ & 32,91 & 35,65 & $-2,74$ & $-7,7 \%$ & \\
\hline Dairygold & $\mathrm{IE}$ & 32,75 & 34,26 & $-1,51$ & $-4,4 \%$ & \\
\hline Milcobel & $\mathrm{BE}$ & 32,72 & 35,75 & $-3,03$ & $-8,5 \%$ & \\
\hline Dairy Crest (Davidstow) & UK & 32,67 & 32,65 & 0,02 & $0,1 \%$ & $1,0 \%$ \\
\hline Glanbia & $\mathrm{IE}$ & 32,59 & 34,31 & $-1,72$ & $-5,0 \%$ & \\
\hline Kerry Agribusiness & $\mathrm{IE}$ & 32,51 & 34,31 & $-1,80$ & $-5,3 \%$ & \\
\hline Valori medi & & 34,23 & 35,42 & $-1,19$ & $-3,4 \%$ & \\
\hline Emmi & $\mathrm{CH}$ & 48,25 & 51,88 & $-3,64$ & $-7,0 \%$ & $-3,1 \%$ \\
\hline Fonterra & NZ & 29,40 & 31,63 & $-2,24$ & $-7,1 \%$ & $0,3 \%$ \\
\hline USA class III & US & 31,49 & 36,22 & $-4,73$ & $-13,1 \%$ & $-8,4 \%$ \\
\hline
\end{tabular}

Fonte: LTO International Milk Price Comparison (5) 


\section{Tabella III. Incidenza dei bonus e delle detrazioni sulla formazione del prezzo del latte}

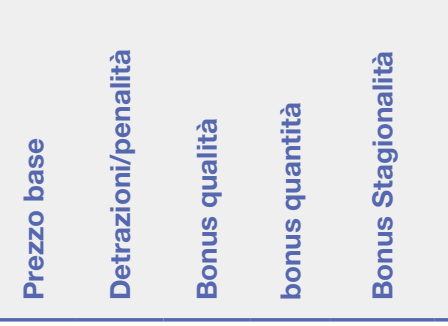

\begin{tabular}{|c|c|c|c|c|c|c|c|c|c|c|}
\hline Granarolo & 37,59 & & 0,77 & & & & & 38,36 & & 38,36 \\
\hline Valio & 34,75 & & & & & 0,97 & & 35,72 & 1,02 & 36,74 \\
\hline $\begin{array}{l}\text { Friesland- } \\
\text { Campina }\end{array}$ & 34,99 & $-0,12$ & & & & 0,63 & & 35,50 & 0,57 & 36,07 \\
\hline Arla Foods DK & 30,87 & $-1,45$ & 0,94 & 0,00 & & 1,00 & 1,82 & 33,18 & 2,30 & 35,48 \\
\hline Royal A-ware & 33,81 & $-1,90$ & 0,50 & 1,28 & & 1,44 & & 35,13 & & 35,13 \\
\hline Sodiaal & 33,81 & $-0,06$ & 0,58 & & & & 0,31 & 34,64 & 0,29 & 34,93 \\
\hline Danone & 34,58 & $-0,03$ & & & & & & 34,55 & & 34,55 \\
\hline Lactalis & 33,44 & & & 0,39 & & 0,15 & & 33,98 & & 33,98 \\
\hline Hochwald & 32,47 & $-0,02$ & 0,76 & 0,51 & & & & 33,72 & 0,02 & 33,74 \\
\hline Savencia & 32,39 & & 1,18 & & & & 0,12 & 33,69 & & 33,69 \\
\hline Müller & 30,98 & & 0,71 & 0,36 & & & 0,30 & 32,35 & 0,67 & 33,02 \\
\hline DMK & 32,23 & $-0,11$ & & 0,20 & & 0,59 & & 32,91 & & 32,91 \\
\hline Dairygold & 36,01 & $-3,88$ & 0,39 & & 0,13 & 0,10 & & 32,75 & & 32,75 \\
\hline Milcobel & 30,76 & $-0,14$ & 0,73 & 1,13 & & & & 32,48 & 0,24 & 32,72 \\
\hline Dairy Crest & 25,42 & & 0,11 & 0,66 & 0,00 & & 6,48 & 32,67 & & 32,67 \\
\hline Glanbia & 34,74 & $-3,88$ & & & 0,24 & & 1,09 & 32,19 & 0,4 & 32,59 \\
\hline Kerry & 36,29 & $-3,88$ & & & & 0,10 & & 32,51 & & 32,51 \\
\hline MEDIA & 33,24 & & & & & & & 33,90 & & 34,23 \\
\hline Fonterra & NZ & 29,40 & 31,63 & $-2,24$ & $-7,1 \%$ & $0,3 \%$ & & & & \\
\hline USA class III & US & 31,49 & 36,22 & $-4,73$ & $-13,1 \%$ & $-8,4 \%$ & & & & \\
\hline
\end{tabular}

Fonte: LTO International Milk Price Comparison (5) funzione della destinazione. Per esempio, il latte di Classe I riguarda il latte che verrà venduto per il consumo liquido, mentre il latte di Classe II verrà destinato alla produzione di formaggi.

Nel presente studio si è deciso di utilizzare il prezzo del latte di Classe III perché una parte rilevante del latte prodotto negli Usa viene destinato alla produzione di formaggi, come avviene anche in Europa, e per questa ragione i prezzi del prodotto sono maggiormente confrontabili. II prezzo reale del latte pagato ai produttori è in genere del $5-10 \%$ più alto di quello definito e pubblicato per la Classe III perché si tratta di un prezzo minimo a cui poi andranno aggiunti i premi di qualità del prodotto e anche perché l'allevatore in molti casi riceve un prezzo che è dato da un mix di classi di prodotti venduti delle diverse Classi quotate nel FMMO.

\section{RISULTATI}

In questo articolo vengono riportati i risultati delle analisi relativi al 2018 confrontate con i risultati ottenuti nel 2017.

In Tabella II sono stati riportati i risultati medi annui dei prezzi calcolati mensilmente in base alla metodologia e ai sistemi di bonus e penalizzazioni descritte. Quindi i valori sono espressi in $€$ per 100 $\mathrm{kg}$ di latte standard. II latte standard viene inteso con $4,2 \%$ grasso e 3,4\% di proteine, una quantità consegnata di 1 milioni di kg annui, una carica batterica di 24.999 e un valore di cellule somatiche di 249.999. I dati sono IVA esclusa. I risultati sono ordinati in ordine decrescente. Per il 2018 il prezzo pagato da Granarolo/Granlatte ai propri conferenti è risultato il più elevato in Europa, seguito dalla finlandese Valio a dalla cooperativa Olandese FrieslandCampina. II risultato di classifica per Granarolo/Granlatte è uguale al 2017 quando al secondo posto si trovava FrislandCampina seguita dalla Royal A-ware sempre olandese.

II valore medio di prezzo calcolato per il 2018 è stato di 34,23 €/100 kg di latte contro i 35,42 €/100 kg di latte calcolati nel 2017. In Tabella III sono stati riportati i valori calcolati per le singole latterie in relazione alla quantificazione delle detrazioni e dei bonus che sono stati sommati al prezzo di base calcolato dalle diverse latterie con il metodo del volume e la correzione in base al grasso e proteine, oppure in base al metodo del latte solido basato solo sui contenuti del grasso e proteine del latte. Dalla somma delle diverse voci si ottiene il subtotale a cui viene aggiunto, quando presente, il conguaglio di fine anno. Considerando solo i valori medi calcolati, è possibile affermare che i bonus e detrazioni hanno inciso in media nelle aziente interessate per $0,66 € / 100 \mathrm{~kg}$ di latte mentre i conguagli di fine anno incidono per un ulteriore $0,32 € / 100 \mathrm{~kg}$ di latte. 


\section{DISCUSSIONE}

Dall'analisi dei risultati è possibile sottolineare alcuni elementi di rilievo.

Nello stesso anno e in Paesi operanti nello stesso ambiente economico/commerciale come l'Unione Europea esiste una enorme variazione dei prezzi pagati agli allevatori che sono andati da $32,51 €$ in Irlanda ai 38,36 € pagati in Italia per la stessa quantità di latte prodotto. Si tratta di una differenza non trascurabile di 5,85 €/100 kg di latte in valore assoluto pari a una differenza del $18 \%$.

II modello organizzativo (Cooperativa o Industria Privata) non sono in stretta correlazione con il livello di prezzo pagato. Se è vero che nel 2018 i prezzi più elevati sono stati pagati da cooperative è anche vero che all'interno della stessa classifica vi sono cooperative che hanno pagato il latte ai propri conferenti un prezzo inferiore a quello pagato da Latterie private.
Il mercato del latte si dimostra ancora una volta un mercato volatile e le variazioni dei prezzi possono essere significative da un anno a quello successivo. Se osserviamo che la variazione media annua tra il 2018 e il 2017 sia stata di 1,19 € pari al 3,4\% le oscillazioni di prezzo per alcune industrie lattiero casearie possono essere molto più rilevanti come ad esempio è accaduto per Milcobel (- 8,5\%) o per DMK con un calo del $7,7 \%$ nell'arco di un solo anno. In altre industrie i prezzi sono rimasti molto più stabili con oscillazioni prossime allo zero virgola, come nel caso di Valio, Dairy Crest e Danone. Queste variazioni possono essere fortemente influenzate da dinamiche interne alle diverse industrie che possono decidere di destinare in particolari anni parte degli utili alle riserve oppure alla remunerazione dei soci.

Le variazioni dei prezzi sul mercato mondiale negli Stati Uniti e in Nuova Zelanda influenzano in modo significativo le variazioni di prezzo sul mercato europeo

\section{CONCLUSIONI}

Il tema della trasparenza dei mercati agricoli è stato da sempre un elemento cruciale e necessario per equilibrare la domanda e l'offerta. II presente progetto milk price comparison ha cercato negli ultimi 20 anni di aumentare la trasparenza del mercato del latte in Europa.

Per far questo è stata creata una metodologia standard che avesse come riferimento i produttori di latte e le fatture da loro emesse per la vendita del latte alle maggiori latterie operanti in Europa.

Le modalità di definizione del prezzo del latte possono variare da latteria a latteria ma in genere sono riconducibili a due categorie:

un calcolo basato sul volume del prodotto e il contenuto \% di grasso e proteine

un calcolo basato sul contenuto in peso del grasso e proteine (latte solido)

A questo prezzo di base vanno poi ad applicarsi una serie di correttivi che nel tempo sono diventati sempre più numerosi creando degli aggravi burocratici non trascurabili all'interno degli allevamenti sempre più soggetti a controlli e verifiche. Infatti, oltre alla composizione del latte hanno acquisito importanza altri parametri di qualità come la carica batterica, le cellule somatiche, e gli sporigeni. Ad essi poi si sono aggiunti altri parametri più generali relativi al rispetto dell'ambiente, al benessere animale, e alla sostenibilità della produzione in termini sempre più ampi.

Non bisogna poi dimenticare le sollecitazioni emerse dal mondo dei consumatori che hanno spinto le industrie lattiero casearie a creare delle linee di prodotto dedicate a rispondere a determinate esigenze. Ne sono esempio le crescenti produzioni biologiche, le linee di prodotto OGM free, le produzioni ad alto contenuto di benessere animale, le produzioni A2A2, ecc.

Oltre a queste linee di prodotto specifiche si affermano sempre di più dei disciplinari "sostenibilità" che cercano di aumentare in modo sostenibile la produzione di latte a 360 gradi.

In queste evoluzioni che riguardano le strategie nazionali o di industrie private che condizionano quindi la produzione del latte negli allevamenti rimangono molto marcate le differenze di prezzo tra i diversi Paesi dell'Unione Europea che a loro volta devono far fronte alla volatilità dei prezzi a livello mondiale.

\section{BIBLIOGRAFIA}

1. Cournot A. Principes de la théorie des richesses, 1863.

2. Walras L. Éléments d'économie politique pure, ou théorie de la richesse sociale, 1874.

3. Varian H.L. Microeconomia (trad it.) Cafoscarina, Venezia, 1987.
4. Saccomandi V. Istituzioni di Economia del Mercato dei Prodotti Agricoli. REDA Edizioni per L'Agricoltura 1991.

5. KOOPS W. LTO International Comparison of Producers Price for Milk 2018 - LTO Nederland 2018. 